Foundations of Physics manuscript No.

(will be inserted by the editor)

David T. Pegg

\title{
Retrocausality and Quantum Measurement
}

Received: date / Accepted: date

\begin{abstract}
A retrocausal interpretation of quantum mechanics is examined and is applied to the problem of measuring an optical qubit before the qubit is actually created. Although the predictions of the retrocausal interpretation are the same as for the conventional causal picture, it provides a new perspective which should give a useful way of understanding some quantum mechanical processes.
\end{abstract}

Keywords quantum mechanics - causality - quantum measurement · retrocausation

PACS 03.65.-w $\cdot 03.65 . \mathrm{Ta} \cdot 03.67 . \mathrm{Lx}$

\section{INTRODUCTION}

Time asymmetry in nature is readily apparent. The arrow of time is manifest in our ability to remember the past but not the future and in the fact that we feel we have some control over future events but not over past events. The latter phenomenon is usually referred to as the principle of causality. Other expressions of causality include the impossibility, in any inertial reference frame, of one observer sending a controllable message that can be received by another observer at an earlier time, that is, the impossibility for information to be sent from one observer to another faster than light. In common with some other authors [1], we shall refer to this expression as the weak principle of causality. A stronger statement than this is that a cause must always

D. T. Pegg

Centre for Quantum Computer Technology, School of Biomolecular and Physical Sciences, Griffith University, Nathan, Brisbane, 4111 Australia

Tel.: +61 737357152

Fax: + 61737357656

E-mail: D.Pegg@griffith.edu.au 
precede its effects, even where this is not necessary for preservation of the weak principle. This statement is essentially what Cramer [1] refers to as the strong principle of causality. A detailed discussion of weak and strong causality has been given by Cramer [1] in terms of what he defines as macroscopic causes and effects. A macroscopic cause is defined as one that can be initiated by an observer and a macroscopic effect is one that allows an observer to receive information. In these terms the weak principle can be restated as the requirement for a macroscopic cause always to precede its macroscopic effects in any reference frame. This clearly restricts observer-observer communication. The strong principle relates to all causes and effects, not just macroscopic ones. Inasmuch as experimental testing involves observers having information about a physical system at different times, only the weak principle can be verified experimentally.

An excellent illustration of the difference between the weak and strong principles of causality can be given in terms of the difference between conventional classical electromagnetic theory and the Wheeler-Feynman absorber theory of radiation [1-3]. The conventional theory, like most physical theories, is formulated in terms of the strong principle, which leads to the retention of only the retarded potential solution of Maxwells equations. In this theory, it is the retarded action of an accelerated charge on itself that causes the charge to lose energy while radiating. In absorber theory, on the other hand, when the retarded radiation from an oscillating charge is absorbed at a later time, it is the advanced radiation from the induced motion of the particles of the absorber at this later time that gives rise to the radiative reaction force, which in turn causes the removal of the appropriate amount of energy from the emitter at the earlier time of oscillation. Although absorber theory contravenes the strong principle of causality, in a universe with perfect absorbing properties it does not violate the weak principle and predicts precisely the same experimentally verifiable results as the conventional, fully retarded, theory of electrodynamics. Thus, just as conventional electrodynamic theory does not allow a controllable message to be sent into the past, neither does absorber theory with the appropriate boundary conditions, even though the latter theory is retrocausal in the sense of contravening the strong principle of causality.

Another theory that can be expressed in terms of both a fully causal picture and a picture that violates the strong principle of causality but not the weak principle is quantum mechanics, which is of relevance to this paper. Adopting a picture that abandons strict adherence to strong causality makes some problems much easier to solve. An example of this is the retrodiction problem in quantum communication in which Bob, by performing a measurement on a quantum system, has to retrodict the state in which Alice prepared the system before transmitting it to him. While this problem can be solved in the conventional picture, it is much simpler to use a retrodictive picture involving a state propagating backwards in time [4-9]. Here the state of a system between the preparation and measurement events is determined by the later measurement event rather than the earlier preparation event. The concept of backward evolving quantum states is also helpful for studying and predicting other interesting quantum phenomena that are not obviously ap- 
parent when the conventional point of view is used (see, for example, Ref. $[10,11]$ and the references therein).

The quantum picture that is retrocausal in the sense of violating the strong but not the weak principle of causality can be extended beyond the use of a state evolving backwards in time for retrodictive purposes. In this paper we use a retrocausal quantum picture to examine the possibility of preparing a qubit state evolving forwards in time in the normal manner, then sending this state into the past where it appears as an identical forward evolving qubit state that can be measured in the normal manner or used for other purposes. We see that this can only be done on a probabilistic basis that does not violate weak causality but it is still useful for the main application described in this paper, that of measuring a quantum optical qubit state before it is prepared. Also, if the qubit sent into the past is used as the input for a quantum computer or a quantum simulator, we obtain an interesting and reasonably simple retrocausal interpretation of a recent proposal to achieve instantaneous quantum computing based on the conventional causal picture suggested by Brukner et al. [12].

We should emphasise from the outset that the retrocausal quantum picture used in this paper is not a new theory of quantum mechanics: it is simply a different interpretation that leads to the same experimental results. Physical processes that are described in the retrocausal picture will also have an interpretation in the conventional picture, which may be quite different. The physical procedure for sending a qubit back into the past that results from the use of the retrocausal picture in Sect. 3 is just that which would be described as the teleportation protocol without correction in the conventional picture. It is thus not a new protocol as such. Retrocausal analysis, however, does provide a different interpretation that can give new intuition for solving various problems and for understanding some quantum processes.

\section{RETROCAUSAL QUANTUM PICTURE}

In the archetypal quantum mechanical experiment, a system is prepared, allowed to evolve and then measured at a later time. Recently [13,14] we have shown that the arrow of time, in the form of weak causality, is inserted into quantum mechanics by the asymmetry in choice of normalization conditions for the non-negative definite operators $\hat{\rho}_{i}$ and $\hat{\Pi}_{j}$ associated with the preparation and measurement events $i$ and $j$ respectively. These normalization conditions are that the trace of $\hat{\rho}_{i}$ is unity and that the sum of $\hat{\Pi}_{j}$ over all possible measurement outcomes $j$ is the unit operator. It is the second of these conditions that prohibits information being sent backwards in time $[13,14]$. These weak causality conditions identify $\hat{\rho}_{i}$ as a density operator and $\hat{\Pi}_{j}$ as an element of a probability operator measure (POM) [15]. In the conventional picture, the state represented by the density operator $\hat{\rho}_{i}$ prepared at time $t_{p}$ evolves to $\hat{U}\left(t_{m}, t_{p}\right) \hat{\rho}_{i} \hat{U}^{\dagger}\left(t_{m}, t_{p}\right)$ at a later time $t_{m}$ when it is measured, where $\hat{U}\left(t_{m}, t_{p}\right)$ is the unitary forward-time evolution operator calculated from the Schrödinger time-dependent equation. The probability 
for a measurement outcome $j$ given a preparation event $i$ is then given by

$$
P(j \mid i)=\operatorname{Tr}\left[\hat{U}\left(t_{m}, t_{p}\right) \hat{\rho}_{i} \hat{U}^{\dagger}\left(t_{m}, t_{p}\right) \hat{\Pi}_{j}\right]
$$

where $\mathrm{Tr}$ is the trace. These predicted probabilities are verifiable experimentally. In this picture, the state of the system between the preparation and measurement times is determined, or caused, by the earlier preparation event and is independent of the later measurement event. This is in line with the strong principle of causality. The experimentally verifiable expression (1), however, can also be written equivalently as

$$
P(j \mid i)=\operatorname{Tr}\left[\hat{\rho}_{i} \hat{U}\left(t_{p}, t_{m}\right) \hat{\Pi}_{j} \hat{U}^{\dagger}\left(t_{p}, t_{m}\right)\right] .
$$

This can be deduced from the cyclic property of the trace or the invariance of the trace under a unitary transformation and the fact that $\hat{U}\left(t_{p}, t_{m}\right)=$ $\hat{U}^{\dagger}\left(t_{m}, t_{p}\right)$. We can interpret this as the measured state propagating backwards in time from the measurement event until it is projected onto the prepared state at the preparation time. (Here we are referring to $\hat{\Pi}_{j}$ as a state. If we wish to reserve this term for a density operator, we can easily obtain a density operator from $\hat{\Pi}_{j}$ by dividing by its trace. This is unnecessary, of course, if $\hat{\Pi}_{j}$ is a pure state projector, which already has unit trace.) In this picture the state of the system between the preparation and measurement times is determined, or caused, by the later measurement event. We shall thus refer to this picture as a retrocausal picture. The retrocausal picture is not in accord with the strong principle of causality. Because of the equivalence of expressions (1) and (2), this retrocausal picture predicts precisely the same measurement probabilities from quantum mechanics as does the conventional picture. Also, we note that, provided we retain the same normalization conditions on $\hat{\rho}_{j}$ and $\hat{\Pi}_{j}$ as in the usual picture, only strong causality is violated; weak causality is maintained and this retrocausal picture does not predict that a controllable message can be sent into the past.

We can regard a quantum picture as being retrocausal, in the sense of violating strong causality, if it involves some states evolving backwards in time, even if it also involves some states evolving forwards in time. A trivial example of this can be seen by rewriting (1) as

$$
P(j \mid i)=\operatorname{Tr}\left[\hat{U}\left(t, t_{p}\right) \hat{\rho}_{i} \hat{U}^{\dagger}\left(t, t_{p}\right) \hat{U}\left(t, t_{m}\right) \hat{\Pi}_{j} \hat{U}^{\dagger}\left(t, t_{m}\right)\right]
$$

where $t_{p}<t<t_{m}$. A more complicated situation is described later.

\section{RETROCAUSAL OPTICS}

An interesting situation arises when the retro-propagating state from the measurement is a two-mode entangled state. The projection of a single-mode forward propagating state onto this state can result in a single-mode retropropagating state carrying some of the characteristics of this forward propagating state. To be specific let us consider the optical case shown in Fig. 1. Ideal photodetectors $D_{0}$ and $D_{1}$ are in output modes $a$ and $b$ of a $50 / 50$ 


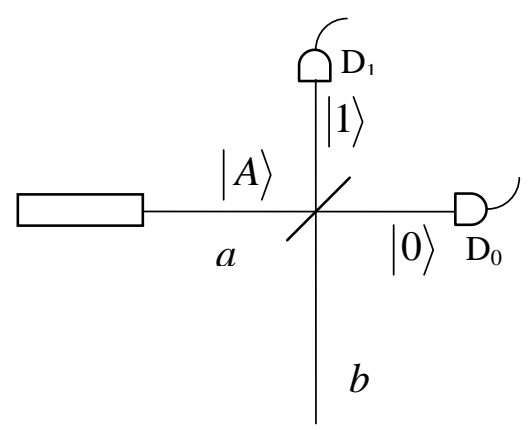

Fig. 1 Ideal photodetectors $D_{0}$ and $D_{1}$ are in output modes $a$ and $b$ of a beam splitter. A field in a forward propagating state $|A\rangle$ is in input mode $a$. The retropropagating state in input mode $b$ arising from the measurement event in which $D_{0}$ and $D_{1}$ register zero photocounts and one photocount respectively will bear some characteristics of $|A\rangle$.

beam splitter. A forward propagating field in the input mode $a$ is in state $|A\rangle_{a}$ at the beam splitter. In the fully causal picture we would consider there also to be a forward propagating field in state $|B\rangle_{b}$, say, in the input mode $b$. Then the probability that $\mathrm{D}_{0}$ and $\mathrm{D}_{1}$ will register zero photocounts and one photocount respectively, that is register the measurement event $(0,1)$, is

$$
\begin{aligned}
P(0,1) & =\left.\left.\right|_{b}\left\langle\left. 1\right|_{a}\left\langle 0\left|\hat{R}_{u}\right| A\right\rangle_{a} \mid B\right\rangle_{b}\right|^{2} \\
& =\left.\left.\right|_{b}\langle B \mid F\rangle_{b}\right|^{2}
\end{aligned}
$$

where $|0\rangle$ and $|1\rangle$ are the vacuum and one-photon number states, $\hat{R}_{u}$ is the forward-time unitary operator describing the action of the beam splitter [16] and

$$
|F\rangle_{b}={ }_{a}\left\langle A\left|\hat{R}_{u}^{\dagger}\right| 0\right\rangle_{a}|1\rangle_{b}
$$

If we regard the input state $|A\rangle_{a}$, the beam splitter and the two photodetectors as constituting a single measuring device, then the POM element for this device representing the outcome that $\mathrm{D}_{0}$ and $\mathrm{D}_{1}$ register the measurement event $(0,1)$ is just $|F\rangle_{b b}\langle F|$. It can be easily checked that the sum of all such elements over all possible photon number outcomes is the unit operator $\hat{1}_{b}$ for mode $b$. From our discussion in Sect. 2 we can regard this as a state propagating backwards in time in the retrocausal picture. An equivalent, but more detailed description in terms of retro-propagating states emanating from the detection events, is as follows. If $D_{0}$ and $D_{1}$ register the measurement event $(0,1)$, the two-mode retro-propagating state in the output modes is $|0\rangle_{a}|1\rangle_{b}$. This state evolves backwards in time to become, on the input side of the beam splitter, the entangled state $\hat{R}_{u}^{\dagger}|0\rangle_{a}|1\rangle_{b}$, at which stage the forward propagating state $|A\rangle_{a}$ is projected onto it to yield the retro-propagating (unnormalized) state $|F\rangle_{b}$ at time $t_{m}$, say, which continues to propagate backwards in time away from the beam splitter as $\hat{U}\left(t, t_{m}\right)|F\rangle_{b}$ where $t<t_{m}$. This state 
carries back with it some characteristics of the forward propagating state $|A\rangle_{a}$.

To be useful, we need to convert the state $\hat{U}\left(t, t_{m}\right)|F\rangle_{b}$ to a forward propagating state at some earlier time $t<t_{m}$. If this can be done, then we should have the retrocausal situation of a forward propagating state with some characteristics of $|A\rangle_{a}$ existing at a time $t$ before $|A\rangle_{a}$ was even prepared. The way of achieving this is just the time inverse of the above: we allow $\hat{U}\left(t, t_{m}\right)|F\rangle_{b}$ to be projected onto a forward propagating two-mode entangled state, resulting in a forward propagating state in the other mode bearing some characteristics of $|A\rangle_{a}$. Now $\hat{U}\left(t, t_{m}\right)|F\rangle_{b}\langle F| \hat{U}^{\dagger}\left(t, t_{m}\right)$ is also an element of a POM, as the sum of all such elements over all photon numbers is $\hat{1}_{b}$. Thus this projection can be regarded simply as a measurement made on mode $b$ of the forward propagating two-mode entangled state at time $t$.

We can combine the above two processes by using the apparatus shown in Fig. 2. The action of $\hat{U}\left(t, t_{m}\right)$ on $|F\rangle_{b}$ is merely to change the phase so, for simplicity, we can replace this unitary operator by the unit operator at the lower beam splitter by making the distance between the beam splitters an integer number of wavelengths. A single photon state and a vacuum state are in the input modes of the lower $50 / 50$ beam splitter. After passing through the beam splitter, they become the entangled state $\hat{R}_{l}|0\rangle_{b}|1\rangle_{c}$ where the subscript $l$ refers to the lower beam splitter. The forward propagating (unnormalized) state $\left|C_{1}\right\rangle$ in output mode $c$ of the lower beam splitter is then just, from (5)

$$
{ }_{b}\left\langle F\left|\hat{R}_{l}\right| 0\right\rangle_{b}|1\rangle_{c}={ }_{b}\left\langle\left. 1\right|_{a}\left\langle 0\left|\hat{R}_{u}\right| A\right\rangle_{a} \hat{R}_{l} \mid 0\right\rangle_{b}|1\rangle_{c} .
$$

This state should bear characteristics of $|A\rangle_{a}$, even though it is produced at an earlier time than $|A\rangle_{a}$. It is not difficult to show for $50 / 50$ beam splitters that [16]

$$
\begin{aligned}
& \hat{R}_{u}^{\dagger}|0\rangle_{a}|1\rangle_{b}=2^{-1 / 2}\left(|0\rangle_{a}|1\rangle_{b}-i|1\rangle_{a}|0\rangle_{b}\right) \\
& \hat{R}_{l}|0\rangle_{b}|1\rangle_{c}=2^{-1 / 2}\left(|0\rangle_{b}|1\rangle_{c}+i|1\rangle_{b}|0\rangle_{c}\right) .
\end{aligned}
$$

By substituting of these expressions into (6) we find that the output state in mode $c$ is given by

$$
\left|C_{1}\right\rangle_{c} \propto{ }_{a}\langle 0 \mid A\rangle_{a}|0\rangle_{c}+{ }_{a}\langle 1 \mid A\rangle_{a}|1\rangle_{c} .
$$

Thus if the input state $|A\rangle$ has the simple two-component form $a_{0}|0\rangle+a_{1}|1\rangle$, the output state $\left|C_{1}\right\rangle$ will have precisely the same form. In this case we have transferred the state of the qubit in mode $a$ to the field in mode $c$ at an earlier time. It is clear that this retrocausality is a purely quantum effect. Classically, no light that is input to the upper beam splitter can possibly reach the lower beam splitter. It is the quantum state that is transferred, not the light itself. For completeness, we note that if the expansion of input state $|A\rangle$ in terms of photon number states $|n\rangle$ contains states with $n>1$, then from (9) these terms are not transferred. This forms the basis of the quantum scissors device $[17,18]$, which gives a means of physically truncating the expansion. Here we are only interested in the retrocausal effect, not the truncation effect, so we shall only be considering the two-component input state $|A\rangle$ above. 


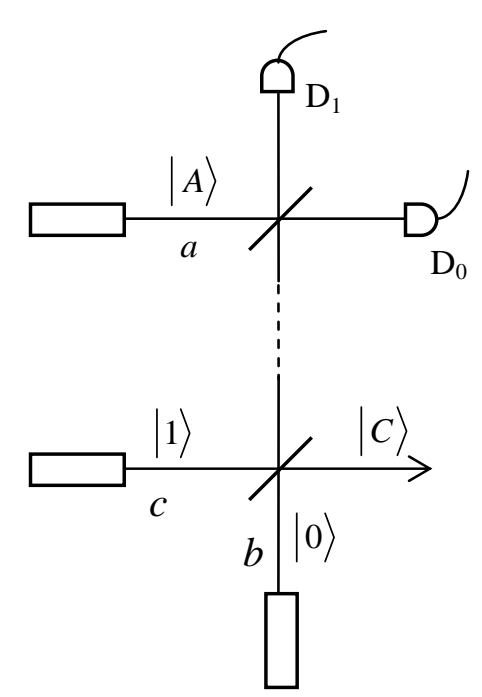

Fig. 2 Device for sending a qubit state back in time. In the retrocausal picture, if photodetectors $D_{0}$ and $D_{1}$ register zero photocounts and one photocount respectively, the qubit state $|A\rangle$ of the input field of the upper beam splitter at time $t_{m}$ is transferred to the output field of the lower beam splitter at the earlier time $t$, that is, the state $|C\rangle$ is the same as $|A\rangle$.

The retrocausal effect described above clearly violates strong causality. It is important to study it also in relation to weak causality. By using the density operator for the state (8) and the POM element $|F\rangle_{b}\langle F| \hat{1}_{c}$, where $\hat{1}_{c}$ is the unit operator for mode $c$, it is not difficult to show that the probability $P(0,1)$ that $\mathrm{D}_{0}$ and $\mathrm{D}_{1}$ will register zero and one photocount respectively is just $1 / 4$. Similarly the probability $P(1,0)$ that $\mathrm{D}_{0}$ and $\mathrm{D}_{1}$ will register one and zero photocounts respectively is also $1 / 4$. In this event the output state $\left|C_{2}\right\rangle_{c}$ in mode $c$ is $a_{0}|0\rangle-a_{1}|1\rangle$. The probability $P(0,0)$ that no photocounts are registered is $\left|a_{0}\right|^{2} / 2$, with the corresponding output state $\left|C_{3}\right\rangle_{c}$ being just $|1\rangle$, and the probability $P(2)$ that a total of two photocounts are recorded is $\left|a_{1}\right|^{2} / 2$, with the corresponding output state $\left|C_{4}\right\rangle_{c}$ being $|0\rangle$. Thus even though we cannot be sure as to which detection event will occur there is an even chance that it will be one of the events $(0,1)$ and $(0,1)$ and in either case some characteristics of the state $|A\rangle$ will be carried by the output state. Thus one might expect that the output state should carry some information about $|A\rangle$. In the absence of any knowledge of the which detection event takes place, however, the best description we can give the output state in mode $c$ is the statistical mixture

$$
\begin{aligned}
\hat{\rho}_{c}= & P(0,1)\left|C_{1}\right\rangle_{c}\left\langle C_{1}|+P(1,0)| C_{2}\right\rangle_{c c}\left\langle C_{2}\right| \\
& +P(0,0)\left|C_{3}\right\rangle_{c}\left\langle C_{3}|+P(2)| C_{4}\right\rangle_{c}\left\langle C_{4}\right| .
\end{aligned}
$$


On substituting the values given above and using $\left|a_{0}\right|^{2}+\left|a_{1}\right|^{2}=1$ we find that terms involving $a_{0}$ and $a_{1}$ vanish, reducing the expression to simply

$$
\hat{\rho}_{c}=\left(|0\rangle_{c}\langle 0|+| 1\rangle_{c}\langle 1|\right) / 2
$$

which contains no information about $|A\rangle$. To obtain information about which detection event occurs we must wait until after the event has taken place, so information about $|A\rangle$ cannot be extracted from $\hat{\rho}_{c}$ before then. Thus controllable information about $|A\rangle$ cannot be transmitted into the past, precluding the sending of a controllable message into the past. Thus, although the above retrocausal effect violates strong causality, it still preserves weak causality.

In this Section we have given an analysis in the retrocausal picture of the experiment depicted in Fig. 2. As mentioned earlier, there will of course be a corresponding analysis in terms of the conventional quantum picture. In the conventional picture, the procedure discussed here for sending a qubit into the past is, in fact, the teleportation protocol without correction, that is, probabilistic teleportation with a probability of $1 / 4$ of success. The two interpretations are equally valid and equivalent. Here we are interested in the retrocausal picture interpretation, rather than what would be seen in the conventional picture as teleportation, as the retrocausal interpretation gives a new intuition for addressing the problem in Sect. 4 .

\section{APPLICATION}

Although we cannot use the above arrangement to send a controllable message into the past, it is nevertheless remarkable that, in this picture, there is one chance in four that a later measurement will register the measurement event $(0,1)$ and confirm that the output state in mode $c$ is $a_{0}|0\rangle+a_{1}|1\rangle$ at a time before the values $a_{0}$ and $a_{1}$ have even been chosen from an infinite number of possibilities. Is there some application that can benefit from this? Let us consider the following, admittedly artificial, simple scenario in order to see how the above retrocausality can be used, at least in principle. For clarity in this example, we shall exaggerate the time interval $t_{m}-t$ to be precisely 24 hours.

Alice is a student and, as an examination, she has to produce an experimental photon-number distribution of an unknown quantum optical qubit $a_{0}|0\rangle+a_{1}|1\rangle$. Bob, her supervisor, is to supply a number of such identical qubits for her to do the experiment. Bob is to supply her with one qubit every minute. Each of her measurements, which will yield the result zero photocounts or one photocount, takes 50 seconds and the result is then automatically sent to a university printer. It is considered that about 60 such measurements are sufficient to obtain a reasonable histogram of the number state distribution. The histogram must be submitted by $5 \mathrm{pm}$ on Friday. It takes the printer, for some reason, 23 hours to print out the results of the measurements on paper and then another one hour for the printer paper to emerge from the printer. Unfortunately Bob is away and cannot be back until 10am on the Friday. What can be done in this situation? The answer is suggested by our previous discussion: Bob uses the device in Fig. 2 to 
send the qubits back 24 hours in time so that Alice starts receiving them in her photodetector in mode $c$ at one per minute from 10am on Thursday. The results start emerging from the printer about 50 seconds after 10 am on Friday at the rate of one per minute. However, Bob successfully sends the correct qubit back only if his photodetectors $\mathrm{D}_{0}$ and $\mathrm{D}_{1}$ register zero and one photocount respectively. This happens approximately one quarter of the time. To rectify this, Bob simply tells Alice on Friday immediately after each attempt at sending back the correct qubit if he has been unsuccessful and she deletes the corresponding result as it emerges from the printer. Alternatively, of course, this correction process could be made automatic. Because only about one-quarter of Bob's attempts to send back the correct qubit will be successful, he sends about 240 qubits back to give Alice the approximate 60 correct results she needs for her histogram. Without the correction process, there would be a string of 240 zeros and ones emerging from the printer in approximately equal numbers. With the correction there are about 60 zeros and ones with the ratio of the number of zeros to the number of ones being $\left|a_{0}\right|^{2} /\left|a_{1}\right|^{2}$.

There is some wasted effort in the above process, this waste being precisely that needed to prevent Bob sending a controllable message back in time. Some effort might be saved if Bob were to tell Alice also to save the printer results when his photodetectors $\mathrm{D}_{0}$ and $\mathrm{D}_{1}$ register one and zero photocounts respectively, because then the qubit $a_{0}|0\rangle-a_{1}|1\rangle$ would have been sent back, which has the same photon number distribution. However, this would not be in accord with the conditions of the examination in which the precise qubit $a_{0}|0\rangle+a_{1}|1\rangle$ must be used. However the process is clearly much more efficient than Alice simply trying to guess the values of $a_{0}$ and $a_{1}$ from an infinite range of possibilities, doing a vast number of experiments and then discarding the incorrect ones after Bob's return.

Because the retrocausal picture must predict the same physical outcomes as the conventional fully causal picture, the conventional picture should lead to the same histogram. In the fully causal picture the starting point is the earlier time $t$. At this time the entangled state (8) is produced in modes $b$ and $c$. The mode $c$ part enters Alice's photodetector for the first measurement and the mode $b$ part evolves until time $t_{m}$, when it enters the upper beam splitter. We have a choice of when the collapse associated with Alice's measurement occurs: when a conscious observer such as Alice views the outcome or when processing by a macroscopic or "classical" device takes place. We examine the former choice first, which gives a situation reminiscent of Schrödingers cat. In this case states $|1\rangle_{c}$ and $|0\rangle_{c}$ in mode $c$ eventually produce states $|1\rangle_{p}$ and $|0\rangle_{p}$ which represent states of the printer output paper in which a one or a zero are shown respectively. At the time just after $t_{m}$ as the entangled field in modes $a$ and $b$ emerges from the upper beam splitter and the printer paper is printed but not yet left the printer to be viewed by Alice, the total state has evolved to

$$
2^{-1 / 2} \hat{R}_{u}|A\rangle_{a}\left(|0\rangle_{b}|1\rangle_{p}+i|1\rangle_{b}|0\rangle_{p}\right) .
$$

On calculating this, we obtain a sum of terms including the term

$$
i 2^{-1}|0\rangle_{a}|1\rangle_{b}\left(a_{0}|0\rangle_{p}+a_{1}|1\rangle_{p}\right) .
$$


with all the other terms being orthogonal to this term. Thus if Bob's detectors $D_{0}$ and $D_{1}$ register zero and one photocount respectively, the state is collapsed to $a_{0}|0\rangle_{p}+a_{1}|1\rangle_{p}$. If $\mathrm{D}_{0}$ and $\mathrm{D}_{1}$ register other than zero and one photocount respectively, the printer result is deleted. At one minute after $t$ the entangled state (8) is again produced, leading to the second printer result one minute after the first, and so on. For each undeleted result the printer paper will be in a superposition state of showing a zero and showing a one. When Alice reads these undeleted results each will collapse either to a zero with probability $\left|a_{0}\right|^{2}$ or to a one with probability $\left|a_{1}\right|^{2}$, giving the same histogram as predicted by the retrocausal picture.

In the other fully causal picture mentioned above, the first collapse is assumed to take place at the earlier time, for example just after $t$ when the field in mode $c$ encounters Alice's photodetector. If this detector registers one or zero photocounts the field in mode $b$ collapses to $|0\rangle_{b}$ or $|1\rangle_{b}$. We can thus calculate the probabilities that Bob's detectors $D_{0}$ and $D_{1}$ register zero and one photocount respectively conditioned on Alice's detector registering one photocount and on it registering a zero count. Using Bayes' theorem we can then calculate the probability that Alice's detector registers one photocount conditioned on $\mathrm{D}_{0}$ and $\mathrm{D}_{1}$ registering zero and one photocount respectively and also the corresponding conditional probability that Alice's detector registers a zero count. Again the same histogram results.

\section{INSTANTANEOUS QUANTUM COMPUTATION}

In Ref. [19] we mentioned that it might be possible, by using an effective retrocausal picture, to provide quantum input into a quantum computer allowing processing to proceed before the required input qubit state is available. An equivalent idea was proposed by Brukner et al. [12] in which the possibility was suggested of using quantum teleportation to perform an entire quantum computation before its quantum input is defined, giving a method for probabilistic instantaneous quantum computing. Here we wish to compare the retrocausal description process in this paper with the causal description of Ref. [12]. We are referring to quantum computing in the broader sense as present quantum computation algorithms use classical inputs and a more appropriate term for the process might be quantum simulation.

We wish to emphasize that the retrocausal description in this paper does not represent a new method for quantum computing, it refers only to the way in which the input state is prepared and not to the quantum computation itself. The computation, or simulation, process itself can be quite standard. To be specific, let us assume that this process involves a series of transformations on $n$ input qubits giving an output that can be a quantum output, or a classical output obtained from the quantum output by a final measurement. The processing time is assumed to be too long for a deadline to be met if the $n$ input qubits cannot be fed in until time $t_{m}$, the time at which Alice is given these qubits. The procedure suggested in [12] to overcome this difficulty and obtain the correct computed result with some non-zero probability of success is as follows. At a time $t$ before $t_{m}$, chosen such that the 
computer processing will finish after $t_{m}$ but within the deadline, Alice prepares $n$ qubits, for example spin-half particles, each of which is maximally entangled with a partner spin. She then feeds these $n$ qubits into the quantum computer and keeps the $n$ partner spins. At the time $t_{m}$ the state of the each entangled pair will have been transformed by an operator that just acts on the qubits in the computer. At this time she is given the correct input qubits. She then immediately performs a Bell-state measurement, that is, a measurement performed in the Bell operator basis [20], on each pair comprising a correct qubit and a spin that she has kept. This is just part of the usual procedure used for quantum teleportation [21]. There is a probability of $1 / 4$ that the outcome of the Bell-state measurement on a pair will be such as to immediately project the associated qubit in the computer onto the state that would have resulted if the correct qubit had been used as an input. Thus there is a probability of $(1 / 4)^{n}$ that the output from the quantum computer will be correct. Although this may be quite small, it is nevertheless better than what can be obtained by random guessing. Also, from the results of the Bell-state measurements, Alice knows whether the output is correct or not and can choose not to accept the wrong output.

In this description, the collapse occurs at the time of the Bell measurement, in a similar way to the first fully causal description given in Sect. 4. It is not difficult to give a retrocausal description instead. In the retrocausal picture the state of the qubit retained by Alice at the earlier time $t$ is determined by the later Bell-state measurement at time $t_{m}$, causing its entangled partner to be in a corresponding state as it enters the computer. There is no collapse of the qubits in the computer after this time. The computer processing proceeds normally and the output produced, quantum or classical, will be that which would be obtained from qubits whose states correspond to the results of the later Bell-state measurement. In the case where the quantum computer processing is finished and, for example, the processing of the classical output has started before the Bell-state measurements take place, this retrocausal description removes the need to consider collapses of Schrödinger cat type superpositions.

\section{CONCLUSION}

In this paper we have used what can be described as a retrocausal analysis of probabilistic teleportation of an optical qubit into the past to examine two particular applications. The first is the case in which optical qubits are created and sent back in time to be measured, with the measurement results being processed before the qubits are created. By retaining only the results for the qubits that are successfully sent back in time, accurate experimental data can be obtained in a time that would be impossible if the measurements were made only after the qubits had been created. The second application is a retrocausal interpretation of the instantaneous quantum computing scheme suggested by Brukner et al. [12], in which quantum teleportation is used to perform a quantum computation before its quantum input qubits are defined. In the causal picture this is achieved by projecting the output state onto the 
correct one with a certain exponentially small but non-vanishing probability. In the retrocausal picture the input qubits are simply sent back in time.

Finally we again stress that the retrocausal picture, which violates the strong principle of causality but not the weak principle, is not a different theory of quantum mechanics from the usual fully causal picture, it is simply a different interpretation. As the retrocausal picture does not violate the weak principle of causality, it cannot be exploited to send a message back in time and is experimentally indistinguishable from the usual picture. However, just as the Schrödinger and Heisenberg pictures provide different perspectives that are useful for treating different problems, the retrocausal picture may also prove useful for some situations, usually where entanglement is involved. Although it is simply a matter of preference as to which picture is used, once the natural caution against violating strong causality can be overcome, the retrocausal picture does have some conceptual advantages.

Acknowledgements I thank Stephen Barnett, John Jeffers and Kenny Pregnell for discussions over the years. This work was supported by the Australian Research Council and the Queensland Government.

\section{References}

1. J. C. Cramer, Phys. Rev. D 22, 362-376 (1980).

2. J. A. Wheeler and R. P. Feynman, Rev. Mod. Phys. 17, 157-181(1945); Rev. Mod. Phys. 21, 425-433 (1949).

3. D. T. Pegg, Rep. Prog. Phys. 38, 1339-1383 (1975).

4. Y. Aharonov, P. G. Bergman, and J. L. Lebowitz, Phys. Rev. 134, B1410-B1416.

5. Y. Aharonov and D. Z. Albert, Phys. Rev. D, 29, 223-227 (1984)

6. Y. Aharonov and D. Z. Albert, Phys. Rev. D, 29, 228-234 (1984)

7. Y. Aharonov and L. Vaidman, J. Phys. A: Math. Gen. 24, 2315-2328 (1991).

8. S. M. Barnett, D. T. Pegg, J. Jeffers, and O. Jedrkiewicz, Phys. Rev. Lett. 86, 2455-2458 (2001).

9. D. T. Pegg, S. M. Barnett, and J. Jeffers, Phys. Rev. A 66, 022106 (2002).

10. Y. Aharonov and L. Vaidman, Phys. Rev. A 41, 11-20 (1990).

11. L. Vaidman, J. Phys. A: Math. Theor. 40, 3275-3284 (2007).

12. C. Brukner, J-W. Pan, C. Simon, G. Weihs, and A. Zeilinger, Phys. Rev. A 67, $034304(2003)$.

13. D. T. Pegg, Phys. Lett. A 349, 411-414 (2006).

14. K. L. Pregnell, Retrodictive Quantum State Engineering. Ph.D. thesis, Griffith University, Brisbane (2004).

15. C. W. Helstrom, Quantum Detection and Estimation Theory (Academic, New York, 1976).

16. S. M. Barnett and P. M. Radmore, Methods in Theoretical Quantum Optics (Oxford University Press, Oxford, 1997).

17. D. T. Pegg, L. S. Phillips, and S. M. Barnett, Phys. Rev. Lett. 81, 1604-1606 (1998).

18. S. A. Babichev, J. Ries, and A. I. Lvovsky, Europhysics Letters 64, 1-7 (2003).

19. S. M. Barnett, J. Jeffers, and D. T. Pegg, "Retrodictive quantum optics," in Coherence and Quantum Optics VIII, N. P. Bigelow, J. H. Eberly, C. R. Stroud, Jr., and I. A. Walmsley, eds. (Kluwer, New York, 2003), pp. 87-94.

20. S. L. Braunstein, A. Mann, and M. Revzen, Phys. Rev. Lett. 68, 3259-3261 (1992).

21. C. H. Bennett, G. Brassard, C. Crépeau, R. Jozca, A. Peres, and W. K. Wooters, Phys. Rev. Lett. 70, 1895-1899 (1993). 